\title{
Поведение доноров лития в объемных монокристаллических моноизотопных сплавах ${ }^{28} \mathrm{Si}_{1-x}{ }^{72} \mathrm{Ge}_{x}$
}

\author{
(C) А.А. Ежевский ${ }^{1}$, П.Г. Сенников ${ }^{2}$, Д.В. Гусейнов ${ }^{1}$, А.В. Сухоруков ${ }^{1}$, \\ Е.А. Калинина ${ }^{1}$, Н.В. Абросимов ${ }^{3}$ \\ ${ }^{1}$ Нижегородский государственный университет им. Н.И. Лобачевского, \\ 603950 Нижний Новгород, Россия \\ ${ }^{2}$ Институт химии высокочистых веществ им. Г.Г. Девятых Российской академии наук, \\ 603950 Нижний Новгород, Россия \\ ${ }^{3}$ Leibniz-Institut für Kristallzüchtung (IKZ), \\ 12489 Berlin, Germany \\ E-mail: ezhevski@phys.unn.ru
}

Поступила в Редакцию 15 апреля 2020 г.

В окончательной редакции 21 апреля 2020 г.

Принята к публикации 21 апреля 2020 г.

Изучено поведение доноров лития в объемных монокристаллических моноизотопных сплавах $\mathrm{Si}_{1-x} \mathrm{Ge}_{x}$ $(x=0.0039-0.05)$, обогащенных бесспиновыми изотопами ${ }^{28} \mathrm{Si}(99.998 \%)$ и ${ }^{72} \mathrm{Ge}(99.984 \%)$ методом электронного парамагнитного резонанса. Исследовалась тонкая структура спектра донорного электрона, локализованного на литии $(T=3.5-30 \mathrm{~K})$, в предположении, что при обогащении бесспиновыми изотопами $\left({ }^{28} \mathrm{Si}^{\text {iso }} \mathrm{Ge}\right.$, iso $\left.=70,72,74,76\right)$ сплавов $\mathrm{Si}_{1-x} \mathrm{Ge}_{x}$ можно достичь, как и в кремнии, более высокого разрешения в спектрах электронного парамагнитного резонанса. Исследования показали, что, несмотря на нерегулярное расположение атомов германия в решетке твердого раствора ${ }^{28} \mathrm{Si}_{1-x}{ }^{72} \mathrm{Ge}_{x}$, создаваемые ими локальные искажения и уширение линий электронного парамагнитного резонанса донорных электронов за счет случайных деформаций в изотопно-чистых монокристаллах ${ }^{28} \mathrm{Si}_{1-x}{ }^{72} \mathrm{Ge}_{x}$ при $x=0.39,1.2,2.9$ ат\%, наблюдались более узкие линии спектров электронного парамагнитного резонанса лития по сравнению с аналогичными кристаллами с природной композицией изотопов кремния и германия, что позволило, как и в кремнии, впервые исследовать угловые зависимости положений линий в спектрах электронного парамагнитного резонанса лития в $\mathrm{Si}_{1-x} \mathrm{Ge}_{x}$ при различных $x$.

Ключевые слова: мелкие доноры, моноизотопные кремний-германий, бесспиновые изотопы, электронный спиновый резонанс, долинно-орбитальное расщепление, электронные состояния, тонкая структура спектра, локальная симметрия, локальные искажения.

DOI: $10.21883 /$ FTP.2020.10.49957.37

\section{1. Введение}

Слабое спин-орбитальное взаимодействие и большие времена спиновой релаксации делают кремний и твердый раствор $\mathrm{SiGe}$ перспективными материалами для квантовых компьютеров [1,2], основанных на спинах электронов, локализованных на донорах. Обогащение матрицы кремния и $\mathrm{SiGe}$ бесспиновыми изотопами приводит к еще более сильной изоляции спинов кубитов от окружения, существенно увеличивая время когерентности спина.

Bсе мелкие доноры V группы в кремнии имеют сходные электронные свойства, определяемые структурой $1 s: A_{1}$ основного состояния электрона - орбитального синглета [3-5]. Тем не менее их спины ведут себя по-разному из-за индивидуальных особенностей $1 s$-состояния, различия в электронной и спиновой плотности на ядре донора и ядрах окружения, а также спин-орбитальной связи, существенно зависящих от индивидуальных химических свойств донорных атомов. Особенности структуры основного состояния доноров можно изучать по спектрам электронного парамагнитного (спинового) резонанса (ЭПР).
Известно, что разрешение спектров ЭПР в кремнии с естественным содержанием изотопов мало изза уширения линий, вызванное изотопным сверхтонким взаимодействием с ядрами ${ }^{29} \mathrm{Si}$ [6]. Это обстоятельство ограничивает возможности анализа экспериментальных спектров.

Обогащение кремния бесспиновым изотопом приводит к существенному сужению линий ЭПР электронов, локализованных на донорах. Такого же эффекта можно ожидать и в сплавах $\mathrm{Si}_{1-x} \mathrm{Ge}_{x}$, избавляясь от изотопов ${ }^{29} \mathrm{Si}$ и ${ }^{73} \mathrm{Ge}[6-8]$. Однако уширение линий ЭПР донорных электронов за счет случайных деформаций, создаваемых растворенными атомами германия в кремнии, может создать конкуренцию уширению магнитными ядрами.

Традиционно остается интерес также к донорным центрам лития, обусловленный тем, что, например, в кремнии, в отличие от доноров V группы, литий является примесью внедрения, располагаясь в тетраэдрическом междоузлии, и обладает инверсной системой уровней $[3,5,9]$. Его синглетный уровень $A_{1}$ находится выше орбитально вырожденных состояний дублета $E$ и триплета $T_{2}$. Эта особенность структуры состояний лития отчетливо проявляется в спектрах ЭПР. Впер- 
вые электронный парамагнитный резонанс электронов, локализованных на изолированных центрах лития в кремнии с природной композицией изотопов (концентрация $N_{\mathrm{Li}} \approx 10^{16} \mathrm{~cm}^{-3}$, температура $T=1.5$ и $\left.4.2 \mathrm{~K}\right)$, был исследован в работах [9-11], а в кристаллах, обогащенных изотопом ${ }^{28} \mathrm{Si}$, - в работах [12-14]. Орбитальное вырождение основного состояния лития, которого нет у доноров V группы, решает очень важную задачу управления спиновыми состояниями квантового бита с помощью импульсов электрической компоненты осциллирующего поля, тем самым воздействуя на спин более локально. В квантовой точке на основе ${ }^{28} \mathrm{Si} / \mathrm{SiGe}$ такого эффекта можно достичь лишь с помощью градиентного поля от наномагнита [15].

Интерес к данным исследованиям вызван еще тем, что сплав $\mathrm{Si}_{1-x} \mathrm{Ge}_{x}$, обогащенный бесспиновыми изотопами $\left({ }^{28} \mathrm{Si}\right.$ iso $\mathrm{Ge}$, iso $\left.=70,72,74,76\right)$, является мало изученным материалом, по сравнению с ${ }^{28} \mathrm{Si}$.

Важным параметром, характеризующим основное состояние донора, является спин-орбитальная связь. Она играет важную роль в явлениях, связанных со спином. В частности, она существенно влияет на процессы спинрешеточной релаксации [16], определяет величину отклонения $g$-фактора донорного электрона от $g$-фактора свободного электрона. Важно знать как решеточные, так и примесные вклады в спин-орбитальные взаимодействия и параметры, которые от них зависят.

Для лития в кремнии с природным содержанием изотопов оценки, сделанные в работе [9] на основе анализа $g$-факторов наблюдаемых спектров, дают высокие значения параметров спин-орбитальной связи $(0.01-0.02) \cdot 1.24 \cdot 10^{-1}$ мэВ, которые выходят за пределы ряда значений, известных для $\mathrm{P}, \mathrm{As}, \mathrm{Sb}, \mathrm{Bi}[16,17]$ $(\Delta \mathrm{so} \approx 0.03$ мэВ для $\mathrm{Si}: \mathrm{P}, 0.1$ мэВ для $\mathrm{Si}: \mathrm{As}, 0.3$ мэВ для $\mathrm{Si}: \mathrm{Sb}$ и 1 мэВ для $\mathrm{Si}: \mathrm{Bi})$. Однако в более поздних работах, выполненных с использованием моноизотопного кремния [12-14], были получены более низкие значения параметров спин-орбитальной связи для изолированного донорного центра лития. В этой связи исследования ЭПР донорных состояний лития в сплавах $\mathrm{SiGe}$, обогащенных бесспиновыми изотопами $\left({ }^{28} \mathrm{Si}{ }^{72} \mathrm{Ge}\right)$, также представляют интерес.

Литий в решетках кремния и сплавов $\mathrm{SiGe}$ подвижен даже при невысоких температурах, поэтому в $\mathrm{SiGe}$ может легко находить энергетически наиболее выгодные места относительно атомов германия, поэтому в зависимости от содержания германия и положения в решетке $\mathrm{Si}_{1-x} \mathrm{Ge}_{x}$ электронная структура основного донорного состояния может меняться. В работе исследовано поведение доноров лития в объемных монокристаллических моноизотопных сплавах $\mathrm{Si}_{1-x} \mathrm{Ge}_{x} \quad(x=0.0039-0.05)$, обогащенных бесспиновыми изотопами. При этом предполагалось, что в сплавах $\mathrm{Si}_{1-x} \mathrm{Ge}_{x}(x=0.0039-0.05)$, так же как и в кремнии, литий является примесью внедрения, располагаясь в тетраэдрическом междоузлии, и обладает инверсной системой уровней по сравнению с донорами V группы.

\section{2. Методика эксперимента}

Монокристаллы ${ }^{28} \mathrm{Si}_{1-x}^{72} \mathrm{Ge}_{x}$ были получены методом Чохральского (Leibniz Institute for Crystal Growth, Berlin, Germany). Легирование фосфором $\left(N_{\mathrm{P}} \approx 5 \cdot 10^{15} \mathrm{~cm}^{-3}\right)$ производилось в процессе роста с использованием в качестве лигатуры тонкого стержня ${ }^{28} \mathrm{Si}$, выращенного методом бестигельной зонной плавки и легированного до концентрации $5 \cdot 10^{17} \mathrm{~cm}^{-3}$ из газовой фазы (в качестве источника фосфора использовался фосфин - $\mathrm{PH}_{3}$ ). Была получена серия образцов с разным содержанием германия и донорной примеси лития и фосфора, обогащенных бесспиновыми изотопами ${ }^{28} \mathrm{Si}(99.998 \%)$ и ${ }^{72} \mathrm{Ge}(99.984 \%)$. Легирование литием проводилось диффузией при $300^{\circ} \mathrm{C}$ в течение 30 мин и фосфором - в процессе роста. Концентрация введенного лития составляла $10^{16} \mathrm{~cm}^{-3}$.

Спектры ЭПР исследовались на спектрометре BrukerEMXplus-10/12 $X$-диапазона в интервале $T=3.5-30 \mathrm{~K}$ (частота 9.6 ГГц). Для измерений угловых зависимостей спектров ЭПР образцы вырезались в виде столбиков $2 \times 2 \times 10$ мм в направлении [011].

\section{3. Результаты и обсуждение}

Несмотря на уширение линий ЭПР донорных электронов за счет случайных деформаций, создаваемых растворенными атомами германия в кремнии, в изотопночистых монокристаллах $\mathrm{Si}_{1-x} \mathrm{Ge}_{x}$, обогащенных бесспиновыми изотопами $\left({ }^{28} \mathrm{Si}(99.998 \%)\right.$ и $\left.{ }^{72} \mathrm{Ge}(99.984 \%)\right)$ при $x=0.39,1.2,2.9$ ат\%, наблюдалось изотопическое сужение линий спектров ЭПР по сравнению с аналогичными кристаллами с природной композицией изотопов кремния и германия вследствие существенного уменьшения вклада в уширение линий, обусловленного сверхтонким взаимодействием с магнитными ядрами ${ }^{29} \mathrm{Si}$ и ${ }^{72} \mathrm{Ge}$. Как видно из рис. 1, это позволило наблюдать спектры электронов, локализованных на донорах фосфора с хорошо разрешенной сверхтонкой структурой (СТC), состоящие из пар линий СТС (наблюдалось четыре различных типа доноров фосфора в $\mathrm{Si}_{1-x} \mathrm{Ge}_{x}$ [18]), и более узкие линии спектров ЭПР, по-видимому, связанных с литием с разрешенной тонкой структурой (рис. 1), отличающиеся локальной симметрией в $\mathrm{Si}_{1-x} \mathrm{Ge}_{x}$ при $x$ больше и меньше $1 \%$. Связь этих линий с литием (центральные линии спектра между линиями СТС фосфора) не вызывает сомнения, так как они появляются только после легирования образцов литием, независимо от того, легировались ли образцы фосфором. При этом в нелегированном при росте кристалле $\mathrm{Si}_{1-x} \mathrm{Ge}_{x}$, (p-типа проводимости), после легирования его литием, мы наблюдали слабые линии фосфора из-за подъема уровня Ферми над уровнем фосфора за счет перекомпенсации остаточного акцепторного бора литием. По сделанным оценкам, концентрация фосфора в таких кристаллах составила $2 \cdot 10^{11} \mathrm{~cm}^{-3}$. Таким образом фосфор ни в 

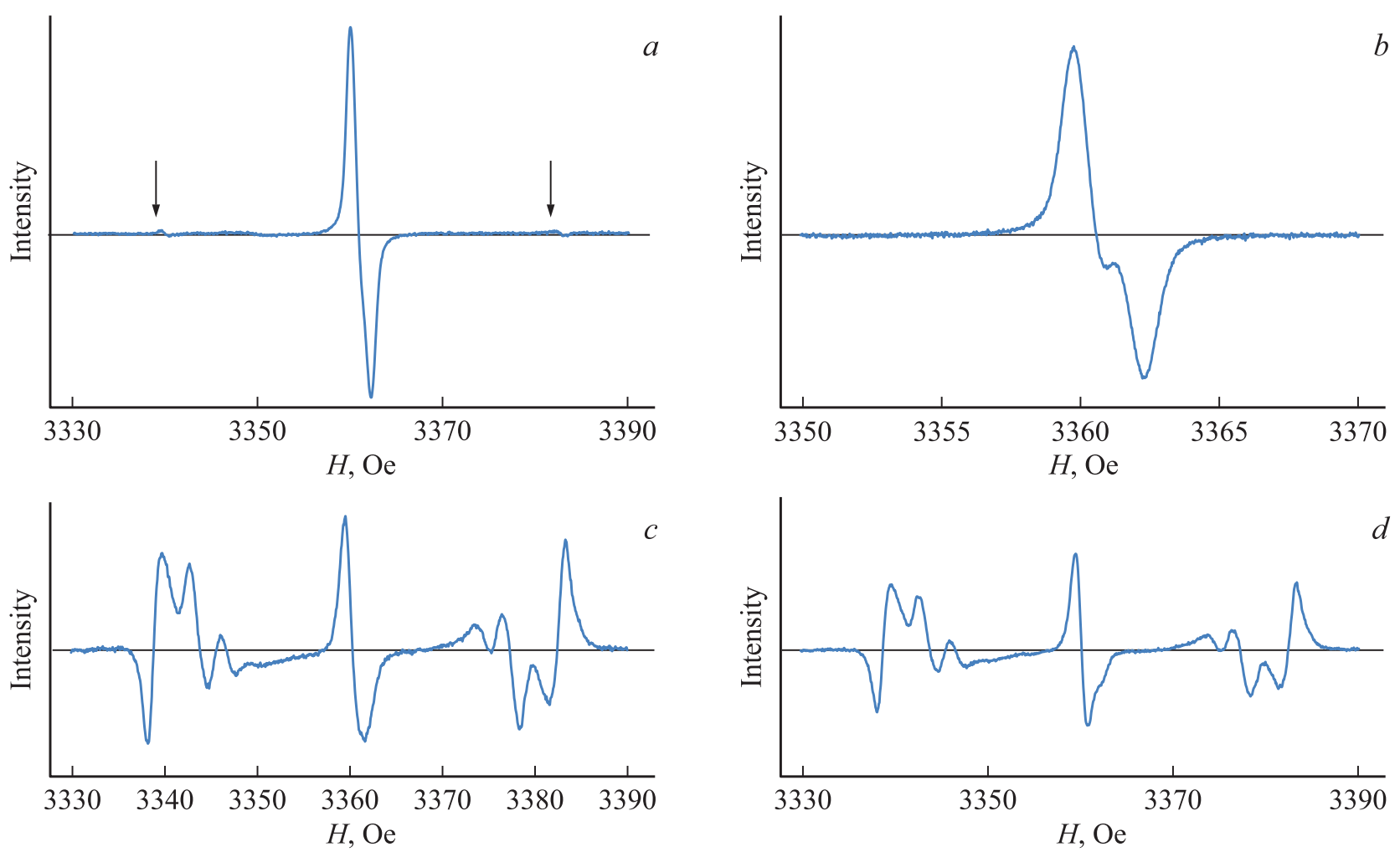

Рис. 1. $a$ - производные сигнала поглощения спектра ЭПР в ${ }^{28} \mathrm{Si}_{1-x}{ }^{72} \mathrm{Ge}_{x}: \mathrm{Li}, x=0.0037, \theta=0^{\circ}(H \|[100])$. Стрелками показаны положения линий фосфора в нелегированном при росте кристалле, расщепленных СТВ; $b-$ то же, при $\theta=90^{\circ}(H \|[011])$; $c$ - производные сигнала поглощения спектра ЭПР в ${ }^{28} \mathrm{Si}_{1-x}{ }^{72} \mathrm{Ge}_{x}: \mathrm{Li}, x=0.011, \theta=0^{\circ}(H \|[100]) ; d-$ то же, при $\theta=90^{\circ}$ $(H \|[011])$.

каких формах не может быть связан с появлением центральных линий спектра. Как видно из рис. 1, у спектров ЭПР для электронов, локализованных на донорных центрах лития в $\mathrm{SiGe}$, отсутствует сверхтонкая структура, что говорит о том, что основное состояние лития, так же как и в кремнии, не является синглетным, в отличие от доноров V группы. Спектры ЭПР фосфора сохраняют изотропию спиновых состояний [18]. Атомы лития, обладая малым радиусом, занимают в $\mathrm{SiGe,} \mathrm{как}$ и в кремнии, междоузлия и имеют, как и в кремнии, орбитально вырожденное основное состояние, проявляя анизотропию $g$-факторов.

Анализ спектров показал, что при $x=0.39 \%$ донорный центр лития обладает аксиальной (тригональной) симметрией с главной осью тензора $g_{\|}(\|[111])=1.9973$ и $g_{\perp}(\perp[111])=1.99805$ (рис. 2). Однако он не связан с известным в кремнии центром LiO [10-14] изза сильного отличия параметров этих спектров и отсутствия сверхтонкой структуры, обусловленной ядрами ${ }^{7} \mathrm{Li}$ [10-12]. При $x \geq 1 \%$ наблюдался спектр, соответствующий тетрагональной симметрии $g$-тензора с $g_{\|}(\|[100])=1.9979$ и $g_{\perp}(\perp[011])=1.9973$ (рис. 3). В этом случае верхняя кривая соответствует положению линии для электрона в состоянии $T_{z}$, а нижняя $T_{x y}$. Такая симметрия наблюдалась для лития в чистом кремнии, где она определялась состояниями $T_{z}$ и $T_{x y}$, образованными с участием кремниевых долин [13,14]. Обнаружение спектра только при $x>1 \%$, возможно, связано с особенностями процессов спин-решеточной релаксации. В рассматриваемых нами кристаллах сплавов $\mathrm{Si}_{1-x} \mathrm{Ge}_{x}$ сохраняется структура долин кремния, поэтому разумно предположить, что тетрагональная симметрия спектра также обусловлена состояниями $T_{z}$

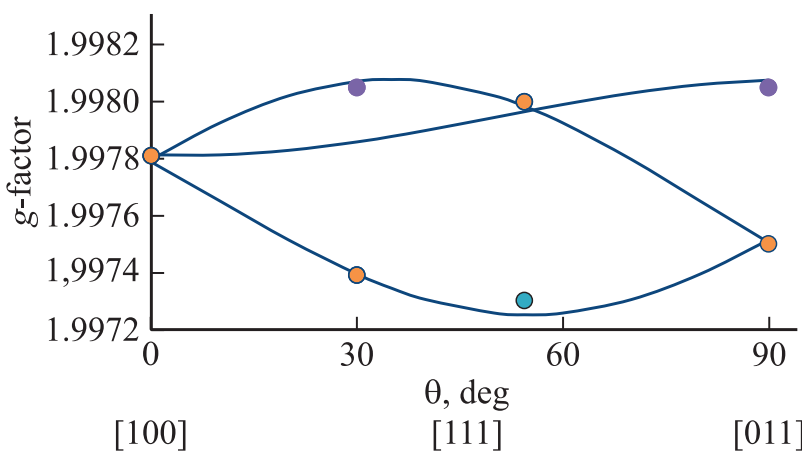

Рис. 2. Угловые зависимости положения линий спектра ЭПР в ${ }^{28} \mathrm{Si}_{1-x}{ }^{72} \mathrm{Ge}_{x}: \mathrm{Li}, x=0.0037$. Точки - экспериментальные данные, сплошные линии показывают вид зависимостей для случая аксиальной (тригональной) симметрии центра в кубическом кристалле с $g_{\|}(\|[111])=1.9973$ и $g_{\perp}(\perp[111])=1.99805$. 


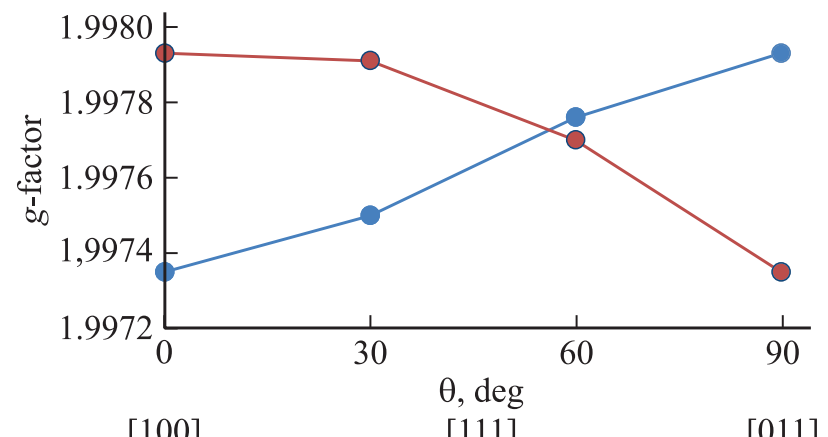

[100]

[111]

[011]

Рис. 3. Угловые зависимости положения линий спектра ЭПР ${ }^{28} \mathrm{Si}_{1-x}{ }^{72} \mathrm{Ge}_{x}: \mathrm{Li}, x=0.011$. Точки - экспериментальные данные, сплошные линии показывают вид зависимостей для случая аксиальной (тетрагональной) симметрии центра в кубическом кристалле с $g_{\|}(\|[100])=1.9979$ и $g_{\perp}(\|[011])=1.9973$.

и $T_{x y}$. Характер угловой зависимости в ${ }^{28} \mathrm{Si}_{1-x}{ }^{72} \mathrm{Ge}_{x}$ несколько отличается от зависимости в чистом кремнии, что, очевидно, связано с вносимыми германием искажениями в решетке $\mathrm{SiGe}$ и, конечно, меньшей точностью определения положения линий из-за большей их ширины по сравнению с кремнием. Характерным является то, что все $g$-факторы оказываются меньше $g$-фактора свободного электрона, как и должно быть для мелких доноров в кремнии и $\mathrm{SiGe}$, и сдвигаются в случае $\mathrm{Si}_{1-x} \mathrm{Ge}_{x}$-сплавов в сторону меньших значений с ростом $x$. Этот результат является важным с точки зрения понимания и оценки вкладов в $g$-факторы со стороны решетки и примеси. Как видно из полученных данных, решеточный вклад от орбитально вырожденных зон приводит к отрицательному сдвигу $g$-фактора, а примесный, от лития, остается таким же малым, как и в кремнии $[13,14]$.

Локальная симметрия спектра лития в ${ }^{28} \mathrm{Si}_{1-x}{ }^{72} \mathrm{Ge}_{x}$ при $x<1 \%$ с главной осью тензора $g_{\|}[111]$ (тригональная симметрия) наблюдается независимо от того, легировались ли они фосфором в процессе роста кристалла. Это указывает на то, что тригональная симметрия спектра лития и, соответственно, связанный с ним парамагнитный центр закономерно возникают при концентрации германия $x<1 \%$. Природу такого поведения лития при $x<1 \%$, по-видимому, еще предстоит более детально исследовать, однако можно предположить, что при очень малом содержании германия в кремнии, когда волновые функции германия еще не перекрываются в решетке кремния, атомы германия ведут себя как изолированные дефектные центры, способные образовывать с литием, обладающим высокой подвижностью и реакционной способностью, изолированные комплексы, типа межузельный литий-германий с осью симметрии [111]. При увеличении содержания германия $x>1 \%$, когда валентные электроны германия встраиваются в валентные состояния решетки кремния, комплексы литий-германий не образуются либо их не видно на фоне преобладающего спектра тетрагональной симметрии от донорных электронов лития (интегральные интенсивности отличаются на порядок), имеющих в этом случае сильно выраженный долинный характер, который определяет симметрию основного состояния донорного электрона лития.

Совокупность полученных результатов свидетельствует о том, что в сплавах ${ }^{28} \mathrm{Si}_{1-x}{ }^{72} \mathrm{Ge}_{x},(x=0.0039-0.05)$ литий, так же как и в кремнии, является примесью внедрения, располагаясь в тетраэдрическом междоузлии, обладает высокой подвижностью и реакционной способностью образовывать комплексы и обладает инверсной системой уровней по сравнению с донорами $\mathrm{V}$ группы. Германий существенно влияет на состояния электронов, локализованных при низких температурах на донорах в ${ }^{28} \mathrm{Si}_{1-x}{ }^{72} \mathrm{Ge}_{x}$, однако при $x<3 \%$ наблюдается изотопическое сужение линий ЭПР из-за уменьшения вклада от сверхтонких взаимодействий с ядрами ${ }^{29} \mathrm{Si}$ и ${ }^{72} \mathrm{Ge}$.

\section{4. Заключение}

Установлено, что несмотря на уширение линий ЭПР донорных электронов за счет случайных деформаций, создаваемых растворенными атомами германия в кремнии, в изотопно-чистых монокристаллах $\mathrm{Si}_{1-x} \mathrm{Ge}_{x}\left({ }^{28} \mathrm{Si}\right.$ $(99.998 \%)$ и и $\left.{ }^{72} \mathrm{Ge}(99.984 \%)\right)$ при $x=0.39,1.2,2.9$ ат\% наблюдались более узкие линии спектров ЭСР лития по сравнению с аналогичными кристаллами с природной композицией изотопов кремния и германия, что позволило наблюдать спектры с разрешенной тонкой структурой, обусловленной анизотропией $g$-факторов. Отсутствие сверхтонкой структуры спектров ЭПР для электронов, локализованных на донорных центрах лития в $\mathrm{SiGe}$, говорит о том, что основное состояние лития в исследованном диапазоне концентраций германия, так же как и в кремнии, не является синглетным, в отличие от доноров V группы, сохраняющих изотропию спиновых характеристик. Атомы лития имеют локальную анизотропию $g$-факторов: тригональную при $x<1 \%$ и искаженную по сравнению с кремнием тетрагональную при $x>1 \%$. Таким образом, германий в ${ }^{28} \mathrm{Si}_{1-x}{ }^{72} \mathrm{Ge}_{x}$ существенно влияет на состояния электронов, локализованных при низких температурах на донорах лития.

\section{Финансирование работы}

Работа выполнена при поддержке гранта РФФИ 18-03-00235-a.

\section{Конфликт интересов}

Авторы заявляют, что у них нет конфликта интересов. 


\section{Список литературы}

[1] M. Veldhorst, H.G.J. Eenink, C.H. Yang, A.S. Dzurak. Nature Commun., 8, 1766 (2017).

[2] F.A. Zwanenburg, A.S. Dzurak, A. Morello, M.Y. Simmons, L.C.L. Hollenberg, G. Klimeck, S. Rogge, S.N. Coppersmith, M.A. Eriksson. Rev. Mod. Phys., 85, 961 (2013).

[3] J.M. Luttinger, W. Kohn. Phys. Rev., 97, 869 (1955).

[4] A.K. Ramdas, S. Rodriguez. Rep. Prog. Phys., 44, 1297 (1981).

[5] A.J. Mayur, M.D. Sciacca, A.K. Ramdas, S. Rodriguez. Phys. Rev. B, 48, 10893 (1993).

[6] V.V. Emtsev Jr., C.A.J. Ammerlaan, A.A. Ezhevskii, A.V. Gusev. Physica B, 376-377, 45 (2006).

[7] D.V. Guseinov, A.A. Ezhevskii, C.A.J. Ammerlaan. Physica B, 381, 164 (2006).

[8] D.V. Guseinov, A.A. Ezhevskii, C.A.J. Ammerlaan. Physica B, 395, 65 (2007).

[9] G.D. Watkins, S.F. Ham. Phys. Rev., 1, 4071 (1970).

[10] A. Honig, A.F. Kip. Phys. Rev., 95, 1986 (1954).

[11] Feher. Phys. Rev., 114, 1219 (1959).

[12] A.A. Ezhevskii, A.V. Soukhorukov, D.V. Guseinov, A.V. Gusev. Physica B: Condens. Matter, 404, 5063 (2009).

[13] A.A. Ezhevskii, S.A. Popkov, A.V. Soukhorukov, D.V. Guseinov, N.V. Abrosimov, H. Riemann. Semiconductors, 46, 1468 (2012).

[14] A.A. Ezhevskii, S.A. Popkov, A.V. Soukhorukov, D.V. Guseinov, A.A. Konakov, N.V. Abrosimov, H. Riemann. Sol. St. Phenomena, 205-206, 191 (2014).

[15] A. Hollmann, T. Struck, V. Langrock, et al. arXiv: 1907.04146v1 [cond-mat.mes-hall] формах (2019)

[16] T.G. Castner. Phys. Rev., 155, 816 (1967).

[17] Y. Song, O. Chalaev, H. Dery. Phys. Rev. Lett., 113, 167201 (2014).

[18] А.А. Ежевский, Д.В. Гусейнов, А.В. Сухоруков, Е.А. Матвеева, Н.В. Абросимов. Тр. XXIV Междунар. симп. „Нанофизика и наноэлектроника“ (Нижний Новгород, 10-13 марта 2020 г.) т. 2, с. 567 (2020).

Редактор А.Н. Смирнов

\section{Behavior of lithium donors in bulk single crystal monoisotopic ${ }^{28} \mathbf{S i}_{1-x}{ }^{72} \mathbf{G e}_{x}$ alloys}

A.A. Ezhevskii ${ }^{1}$, P.G. Sennikov ${ }^{2}$, D.V. Guseinov ${ }^{1}$, A.V. Soukhorukov ${ }^{1}$, E.A. Kalinina ${ }^{1}$, N.V. Abrosimov ${ }^{3}$

${ }^{1}$ Lobachevsky State University of Nizhny Novgorod, 603950 Nizhny Novgorod, Russia

${ }^{2}$ Devyatykh Institute of Chemistry

of High-Purity Substances,

Russian Academy of Sciences,

603950 Nizhny Novgorod, Russia

${ }^{3}$ Leibniz-Institut für Kristallzüchtung (IKZ), 12489 Berlin, Germany

Abstract We studied the behavior of lithium donors in bulk single-crystal single-crystal alloys $\mathrm{Si}_{1-x} \mathrm{Ge}_{x},(x=0.0039-0.05)$, enriched in spinless isotopes ${ }^{28} \mathrm{Si}(99.998 \%)$ and ${ }^{72} \mathrm{Ge}(99.984 \%)$ by electron paramagnetic resonance (EPR). The fine structure of the spectrum of a donor electron localized on lithium $(T=3.5-30 \mathrm{~K})$ was studied under the assumption that, when spinless isotopes $\left({ }^{28} \mathrm{Si}^{\text {iso }} \mathrm{Ge}\right.$, iso $\left.=70,72,74,76\right)$ are enriched in $\mathrm{Si}_{1-x} \mathrm{Ge}_{x}$ alloys, it is possible to achieve in higher resolution in the EPR spectra as was observed in silicon. Studies have shown that despite the irregular arrangement of germanium atoms in the lattice of a $\mathrm{SiGe}$, the local distortions they create and the broadening of the EPR lines of donor electrons due to random deformations in isotopically pure ${ }^{28} \mathrm{Si}_{1-x}{ }^{72} \mathrm{Ge}_{x}$ single crystals at $x=0.39,1.2,2.9 \%$, narrower lines of the ESR spectra of lithium were observed in comparison with similar crystals with a natural composition of silicon and germanium isotopes, which made it possible, as in silicon, to study for the first time the angular dependences of the line positions in the ESR spectra of lithium in $\mathrm{Si}_{1-x} \mathrm{Ge}_{x}$ for various $x$. 